\title{
Correction to: Identification of adenine-N9-(methoxy) ethyl- $\beta$-bisphosphonate as NPP1 inhibitor attenuates NPPase activity in human osteoarthritic chondrocytes
}

\author{
Molhm Nassir ${ }^{1}$ - Uri Arad ${ }^{2}$. Sang-Yong Lee ${ }^{3} \cdot$ Shani Journo ${ }^{2}$. Salahuddin Mirza Christian Renn ${ }^{3}$. \\ Herbert Zimmermann ${ }^{4}$. Julie Pelletier ${ }^{5}$. Jean Sévigny ${ }^{5,6}$. Christa E. Müller ${ }^{3}$. Bilha Fischer ${ }^{1}$
}

Published online: 20 July 2019

(C) Springer Nature B.V. 2019

\section{Correction to: Purinergic Signalling}

https://doi.org/10.1007/s11302-019-09649-2

The original version of the article unfortunately contained an error.

Three authors were not included in the original paper. The complete list is given above.

The first two authors (Molhm Nassir and Uri Arad) are of equal contribution.

The online version of the original article can be found at https://doi.org/ 10.1007/s11302-019-09649-2

Bilha Fischer

bilha.fischer@biu.ac.il

1 Department of Chemistry, Bar-Ilan University,

52900 Ramat-Gan, Israel

2 Department of Rheumatology, Tel Aviv Medical Center and the Faculty of Medicine, Tel Aviv University, 6997801 Tel Aviv, Israel

3 PharmaCenter Bonn, Pharmaceutical Institute, Pharmaceutical Chemistry I, University of Bonn, Bonn, Germany

4 Institute of Cell Biology and Neuroscience, Goethe University, 60438 Frankfurt am Main, Germany

5 Centre de Recherche du CHU de Québec, Université Laval, Québec City, QC, Canada

6 Département de Microbiologie-Infectiologie et d'Immunologie, Faculté de Médecine, Université Laval, Québec City, QC, Canada 